\title{
THE MICHIGAN ION BEAM LABORATORY FOR SURFACE MODIFICATION AND ANALYSIS
}

\author{
G.S. WAS and V.H. ROTBERG \\ Department of Nuclear Engineering. University of Michigan, Ann Arbor, MI 48109, USA
}

The Michigan Ion Beam Laboratory for Surface Modification and Analysis was established in October of 1986 for the purpose of advancing our understanding of ion-solid interactions on both the fundamental and applied levels. The facilities consist of a $1.7 \mathrm{MV}$ Tandetron accelerator, a $2 \mathrm{MV}$ Van de Graaff accelerator and a $400 \mathrm{kV}$ ion implanter. One of the major objectives of the laboratory is to provide an understanding of how ion beam surface modification can be used to improve physical and mechanical properties of engineering materials. As such, several programs have been established to determine the mechanisms by which ion beam surface modification affects properties such as fatigue, corrosion, stress corrosion cracking and wear. These programs provide an interface, or technology transfer, between fundamental aspects of ion-solid interaction and their application to engineering products.

\section{About the Laboratory}

The Michigan Ion Beam Laboratory is part of the Department of Nuclear Engineering in the College of Engineering, and is located on the University of Michigan's North Campus. The laboratory was created for the purpose of advancing our understanding of ion-solid interactions by providing unique and extensive facilities to support research at the cutting edge of science. Researchers have available to them several instruments for conducting ion beam surface modification (implantation, mixing) as well as ion beam surface analysis (RBS, NRA, ion channeling, forward recoil scattering). Experiments can be conducted at high or low temperature, in ultrahigh vacuums and with a short turnaround time. A considerable amount of supporting facilities are also available for surface preparation. The following sections describe the facilities as well as the capabilities of this laboratory and its role in transfer of technology between fundamental aspects of ion-solid interactions and their application to engineering products.

\section{The facilities}

The lab houses a 1.7 MV Tandetron accelerator, a 2 MV Van de Graaff accelerator and a $400 \mathrm{kV}$ ion implanter (fig. 1). Additional major facilities consist of a cryopumped vacuum evaporator equipped with two electron beam guns and two thickness monitors, a 10 MW pulsed ruby laser and a data acquisition system which consists of an Apollo DN4000 and an AT-type computer interfaced with the Tandetron and Van de Graaff accelerators and equipped with two EG\&G plug-in cards which provide emulation software to produce two fully featured MCAs. The capabilities of the accelerators are discussed in the following subsections.

\subsection{The 1.7 MV Tandetron accelerator}

The Tandetron accelerator, made by General Ionex, is a solid-state, gas-insulated, high frequency device capable of operation between 0.2 and $1.7 \mathrm{MV}$ (fig. 2). The accelerator can operate either with a duoplasmatron source or a sputter-type ion source. The duoplasmatron source is a high brightness ion source that produces $\mathrm{H}^{+}$and $\mathrm{He}^{+}$currents of about $1 \mu \mathrm{A}$ at the target. Other gases can also be produced. The sputter ion source can produce ions of many elements at currents ranging from 1 to $5 \mu \mathrm{A}$ at the target. Since a tandem ion accelerator operates by producing negative ions and stripping electrons at the terminal, the available ion energy range extends from about $400 \mathrm{keV}$ to

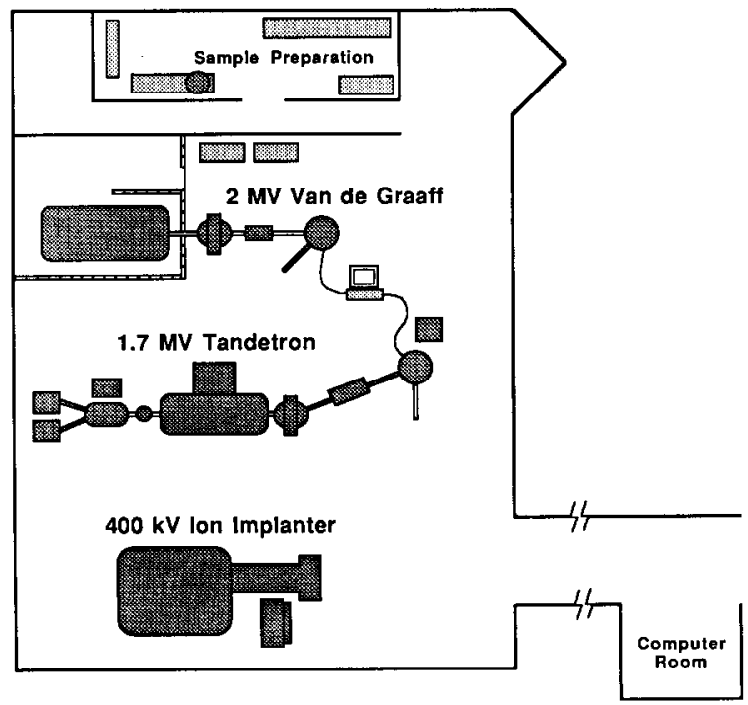

Fig. 1. Layout of the Michigan Ion Beam Laboratory. 


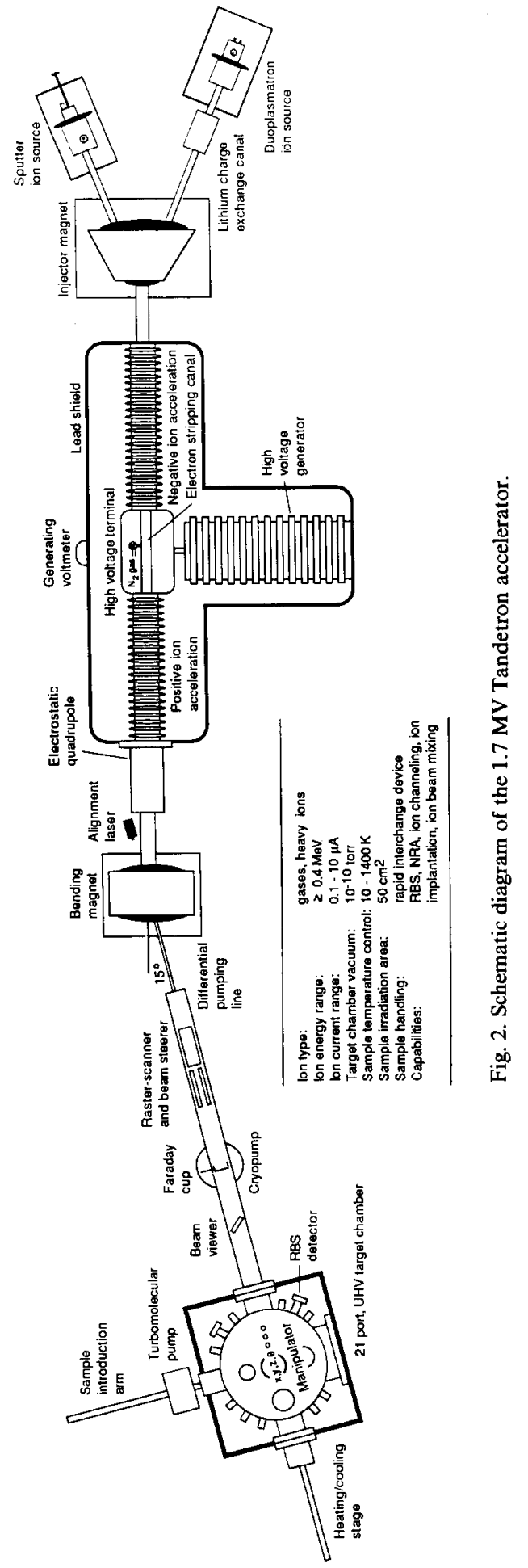

several $\mathrm{MeV}$ (for multiply charged ions), providing an extremely broad energy range to support a variety of experiments. The high energy analysis extension and beamline consist of a quadrupole triplet for focusing, an analyzing magnet, a raster-scanner and steerer system, an aperture system for ion channeling, a beam viewer, and an in-line Faraday cup. The highly versatile target chamber has 20 access ports, an $x, y, z, \theta$ sample manipulation stage and an introduction stage for rapid interchange of large samples. The chamber is also equipped with a heating/cooling stage capable of temperatures for 10 to $1400 \mathrm{~K}$. The beamline and target chamber are made of stainless steel and are pumped with cryopumps providing a true UHV system. The Tandetron accelerator is well suited to surface modification studies using the sputter ion source. Depending on the element, ion currents can be produced which are high enough to reach doses of $1 \times 10^{16}$ over a $1 \mathrm{~cm}^{2}$ area in a few minutes. The duoplasmatron source can be used for Rutherford backscattering spectrometry (RBS), ion channeling or nuclear reaction analysis (NRA).

\subsection{The $2 M V$ Van de Graaff accelerator}

The 2 MV Van de Graaff operates with a radiofrequency ion source and is capable of producing several tens of microamperes of current of both $\mathrm{H}^{+}$and $\mathrm{He}^{+}$. A slit system maintains the beam energy at the preset level. The beamline consists of a magnetic quadrupole for beam focusing, an analyzing magnet, a beam steerer, a beam steerer, a beam viewer and a Faraday cup (fig. 3). The target chamber consists of 18 access ports, an $x$, $y, z, \theta, \phi$ sample manipulator and an introduction stage for rapid sample transfer. The accelerator operates between 0.5 and $2.0 \mathrm{MV}$ and can deliver in excess of $1 \mu \mathrm{A}$ of beam on target. The Van de Graaff has been configured as a dedicated Rutherford backscattering system.

\subsection{The $400 \mathrm{kV}$ ion implanter}

The $400 \mathrm{kV}$ ion implanter, made by Varian, is well suited to low energy, high dose ion implantation of a variety of species using gaseous and solid sources. Ions produced in a cold cathode ion source and vaporizer pass through a $90^{\circ}$ analyzing magnet and are then accelerated. Currents of up to $1 \mathrm{~mA}$ on target are achievable in this machine giving it the capability of high dose $\left(>10^{17} \mathrm{~cm}^{-2}\right)$ implantation in a matter of minutes. The beamline and the stainless-steel chamber are cryopumped to provide a high vacuum system, and a $\mathrm{LN}_{2}$ cooled sample holder and radiant heat source provide in situ sample cooling and heating, respectively (fig. 4). This machine is suitable for implantation into semiconductors, ceramics, metals and polymers. 


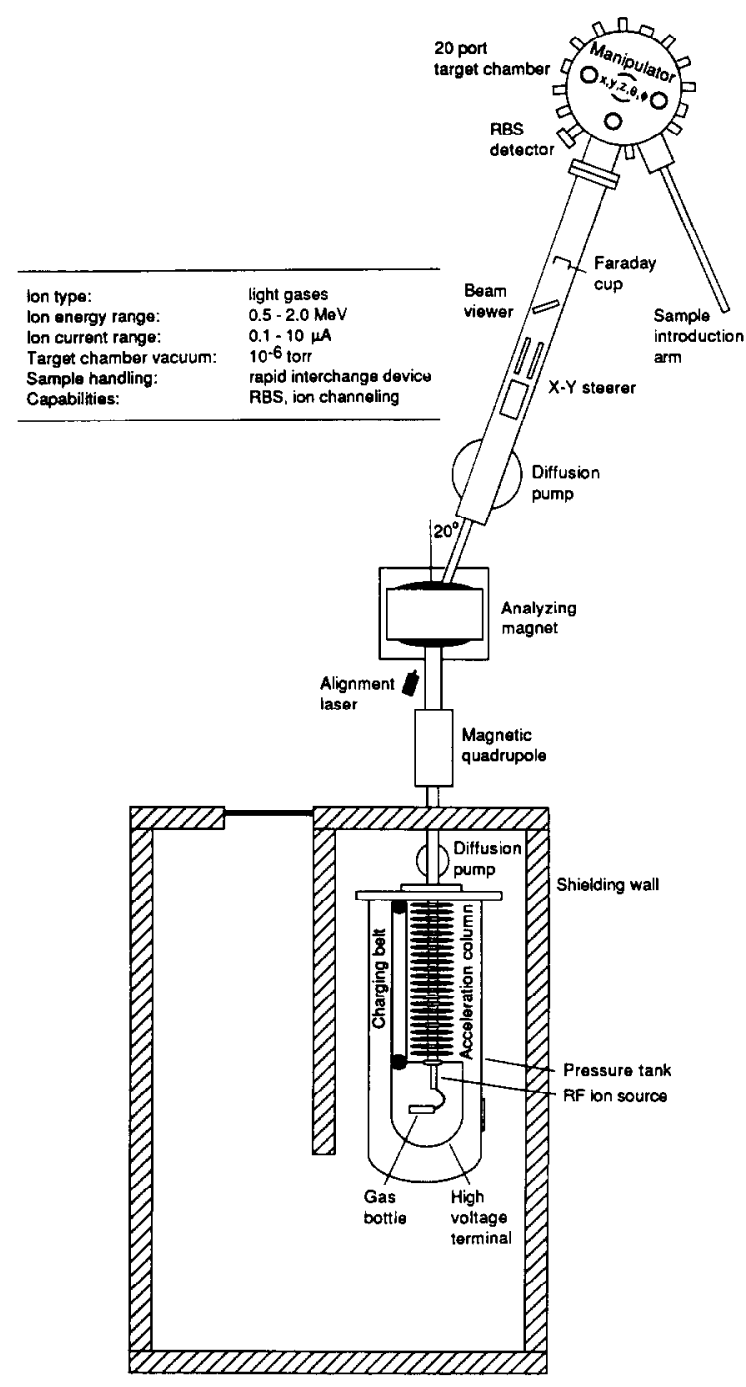

Fig. 3. Schematic diagram of the 2 MV Van de Graaff accelerator.

\subsection{Data acquisition and analysis system}

The data acquisition and analysis system consists of a PC Limited model AT100, AT-type computer with 1 Mbyte RAM and a 20 Mbyte hard disc. The computer is equipped with two EG\&G Ortec ACE plug-in cards which provide emulation software to produce two fully featured MCAs. Separate preamplifiers and amplifiers allow independent and simultancous collection of RBS spectra from the Van de Graaff and the tandem. The system is interfaced with the Michigan Terminal System's mainframe Amdahl computers for large, time-consuming jobs.

The lab also shares an Apollo DN4000 engineering workstation with supermini computer performance. The workstation has 32 Mbytes of RAM, 1 Gbyte of virtual memory, a 200 Mbyte hard disk with type cartridge and a high resolution monochrome monitor. The workstation is networked into about 350 other Apollos as well as an Alliant FX8.

\subsection{Additional facilities}

A dual electron beam evaporator for thin film preparation is also available. The system is equipped with dual electron beam guns providing four source positions and two thickness monitors for rapid preparation of either codeposited or layered films. The evaporator is equipped with a shutter system and the capability of evaporating onto rotating cylindrical samples. A sorption and a cryopump combine to provide an oil-free environment.

\section{Ion beam surface modification}

Both ion implantation and ion beam mixing can be conducted in either the Tandetron or the Varian accelerators. Ion implantation is a non-equilibrium technique in which energetic ions are introduced into the near surface region of solids. The energy of the incoming beam can be controlled to tailor the depth distribution of the implanted species. Current integration provides an accurate measure of implanted species concentration and raster scanning insures spatial uniformity. Ion implantation is particularly well suited to research investigations as well as practical applications such as the modification of friction, wear, corrosion, and fatigue in metals and doping in semiconductors.

In ion beam mixing, heavy ion bombardment of thin film composite materials can be an extremely efficient process for making surface structures which are otherwise impossible to produce. When thin films of two or more elements are alternately deposited on a substrate and bombarded with energetic heavy ions, the elements can intermix by ballistic processes. Ion beam mixing has been utilized to produce novel surface structures ranging from stable compounds to amorphous and metastable structures not achievable by conventional processing techniques. The implanter is used for mixing to depths of 1000-2000 $\AA$ while the tandem ion accelerator has been used to mix micron-thick films.

\section{Surface analysis with ion beams}

Analysis of the number and energy of ions ( $\mathrm{H}$ or $\mathrm{He}$ ) scattered from a solid by the Rutherford elastic scattering process can provide a nondestructive, quantitative, mass-specific depth profile of the first few microns of the surface. Since the energy of the scattered $\mathrm{He}$ depends on the mass of the target atom, separate energy 


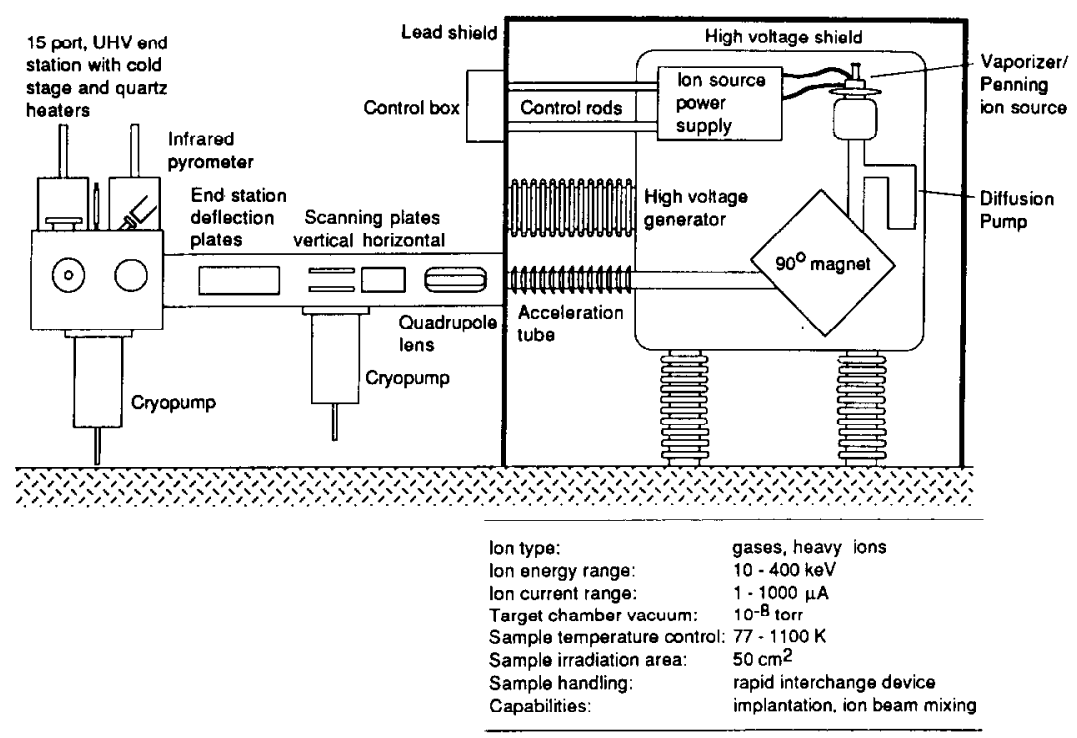

Fig. 4. Schematic diagram of the $400 \mathrm{kV}$ Varian ion implanter.

spectra are obtained from each of the elements in the sample. Because the energy loss processes are well known, the scattered ion energy spectra can be converted into separate depth profiles.

In the example shown in fig. 5 , the as-evaporated sample is characterized by distinct layers of $\mathrm{Ni}$ and $\mathrm{Al}$ separated in energy. Following mixing with an ion beam

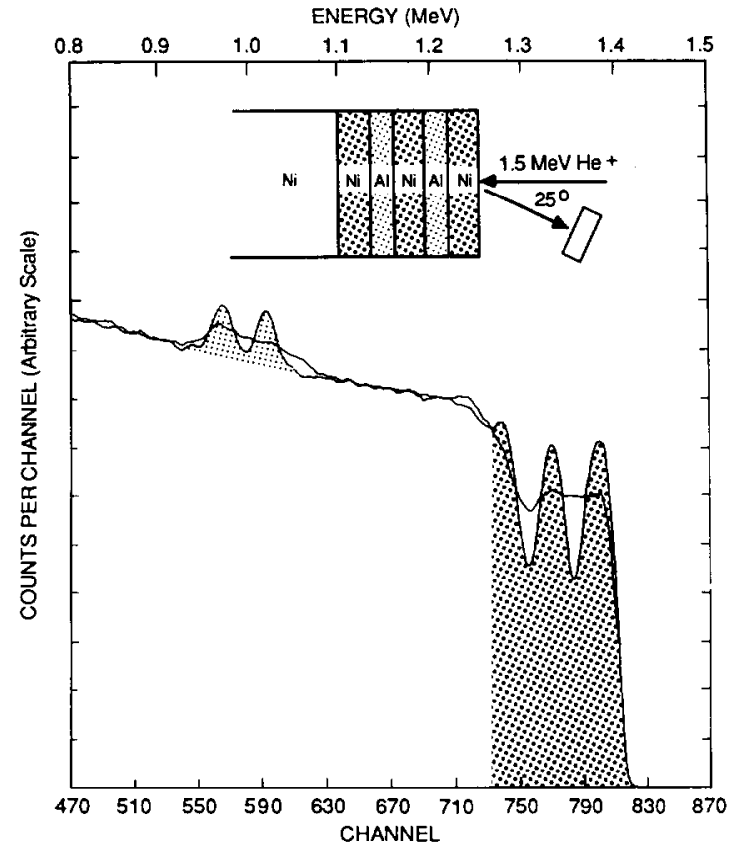

Fig. 5. Rutherford backscattering spectra from a nickel substrate with layers of $\mathrm{Ni}$ and $\mathrm{Al}$ before and after mixing with an ion beam. the surface layer is compositionally homogenized as reflected by the flat RBS profile. The individual layer thicknesses of multilayered samples as well as the thickness of homogeneous films can be determined quickly and accurately using available software. When the host target is a single crystal, the ions can be steered by atomic planes (ion channeling) and the scattered ion energy spectra become altered to provide valuable, additional information. The combination of ion scattering and channeling can be utilized for surface and radiation damage studies, lattice location and diffusion studies.

Forward recoil spectrometry is a method for obtaining depth profiles of light elements (such as $\mathrm{H}$ or $\mathrm{D}$ ) in solids. In this technique, shown in fig. 6 , He ions of a few $\mathrm{MeV}$ are scattered off the target, but instead of

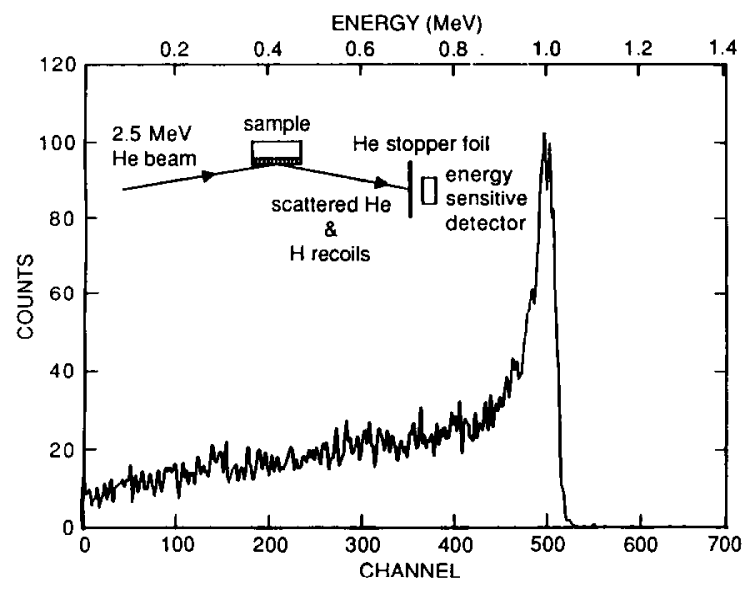

Fig. 6. Forward recoil spectrum of hydrogen contained in the surface layer of the target.

VI. MATERIALS ANALYSIS FACILITIES 


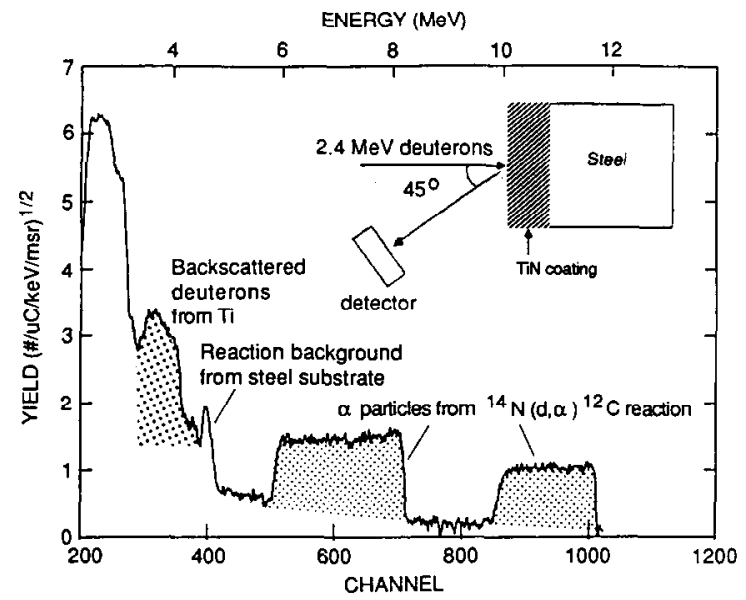

Fig. 7. Nuclear reaction analysis of the stoichiometry of a TiN film using the ${ }^{14} \mathrm{~N}(\mathrm{~d}, \alpha){ }^{12} \mathrm{C}$ reaction from a $2.4 \mathrm{MeV}$ deuteron beam.

measuring the energy of the scattered He particles, the energy of the forward scattered hydrogen is measured. This is accomplished by shielding the detector from $\mathrm{He}$ ion with a thin foil so that only $H$ or $D$ ions reach the detector. Hydrogen and deuterium concentration profiles in solids can be measured to depths of over a micron.

For certain ion-atom combinations nuclear reactions can occur which emit ionizing radiation as reaction products. Since these reactions occur with known probability at definite energies, one can obtain a quantitative measure of the concentration by using the energy dependent reaction cross-section to deconvolute the yield of the reaction product as a function of depth (energy). In the example shown in fig. 7, the stoichiometry of a TiN film is determined by comparing the $\alpha$ yield from the ${ }^{14} \mathrm{~N}(\mathrm{~d}, \alpha){ }^{12} \mathrm{C}$ reaction to that of a standard.

\section{The role of the university in technology transfer}

The development of ion beam technology for modifying and analyzing surfaces of solids has been marked by rapid and dramatic improvements in both implantation techniques and implantation equipment. As this progress has occurred, the application of ion beam modified surfaces in engineering systems has become increasingly evident. Today many industries are experimenting with this technique as a means for improving surface properties of engineering components. Such rapid advances have made it possible to foresee the day when ion beam surface modification becomes an integral part of materials processing in many industries.
The role of the university laboratory in technology transfer is a unique one. The university is neither a private business nor a government agency, and hence can neither fund research nor act as the end recipient of advanced technologies. Therefore, the university laboratory has the opportunity to occupy a unique position in the evolutionary chain of technology transfer. It is not, nor should it be, the university's role to compete with private enterprise for customers and business in ion beam surface modification. However, close ties with industry are beneficial for the university as well as business. The university has the opportunity to capitalize on its position as a research institution to provide business and industry with the resources (personnel as well as facilities) to investigate in depth potential applications of ion beam surface modification - a luxury that the surface modification industry cannot often afford. The method of conducting both basic and applied research at a university lends itself well to projects which require considerable thought, development and testing before successful large-scale implementation of the technique can be assured. Such projects provide ideal opportunities for students and laboratory personnel to become involved in the field. Aside from the benefits derived from the results of the research, perhaps the most important product is the education of the student. The future of any high technology depends critically on the quality and number of individuals who are drawn into it. The students who work on research and development projects for industry are the future of that field.

The Michigan Ion Beam Laboratory has been active in interfacing with industry to provide the understanding needed to apply these techniques to surface processing. The lab has done this in several ways including interaction with individual scientists and engineers in industry as well as the establishment of an industrial affiliates program. The University of Michigan Industrial Affiliates Program in Ion Beam Surface Modification and Analysis was established to provide an organized structure by which improved interaction between industry and the University can occur in the surface modification area. Broadly stated, the Program has as its goals to encourage closer interaction with industry, to provide industry with access to surface modification and analysis facilities, to ensure excellence in University research and teaching in ion beam surface modification and analysis, and to provide assistance to industry in meeting its needs for highly trained personnel. The Program is thus intended as a shared commitment and partnership between the faculty and each industrial affiliates member. 